\title{
Symptom severity, quality of sleep, and treatment adherence among patients suffering from schizophrenia and depression
}

\author{
Peggy Bosch ${ }^{1,2,3}$, Janina Waberg ${ }^{1,2}$, Maurits van den Noort ${ }^{3,4}$, Heike Staudte ${ }^{2}$, Sabina Lim³ ${ }^{3}$, Jos Egger ${ }^{1,5,6,7}$ \\ ${ }^{1}$ Centre for Cognition, Donders Institute for Brain, Cognition and Behaviour, Radboud University Nijmegen, 6500 GL Nijmegen, the \\ Netherlands. \\ ${ }^{2}$ Psychiatric Research Institute, LVR-Klinik Bedburg-Hau, 47511 Bedburg-Hau, Germany. \\ ${ }^{3}$ Research Group of Pain and Neuroscience, Kyung Hee University, Seoul 130-701, South Korea. \\ ${ }^{4}$ Brussels Institute for Applied Linguistics, Vrije Universiteit Brussel, 1050 Brussels, Belgium. \\ ${ }^{5}$ Behavioural Science Institute, Radboud University Nijmegen, 6500 GL Nijmegen, the Netherlands. \\ ${ }^{6}$ Centre of Excellence for Neuropsychiatry, Vincent van Gogh Institute for Psychiatry, 5803 AC Venray, the Netherlands. \\ ${ }^{7}$ Pompe Institute for Forensic Psychiatry, PRO Persona, 6503 CK Nijmegen, the Netherlands.
}

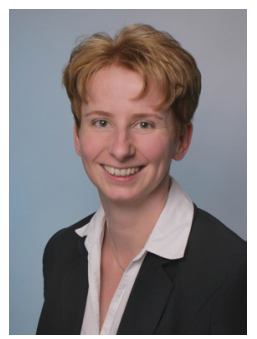

Dr. Peggy Bosch received her PhD from the Radboud University, Nijmegen in the Netherlands and works as a clinical psychologist for the LVR-Klinik Bedburg-Hau in Germany. She is especially interested in schizophrenia, depression and sleep disorders.

\begin{abstract}
Aim: Treatment non-adherence is a common problem in patients suffering from schizophrenia and depression. This study investigated the possible relationships between symptom severity, quality of sleep, and treatment adherence. Methods: Thirty outpatients with schizophrenia and 58 outpatients with depression were enrolled in this study. The beck depression Inventory-II, the positive and negative syndrome scale, and the pittsburgh sleep quality index were used to assess symptom severity and quality of sleep, and sleep log data were used to measure treatment adherence. Results: The preliminary results showed no significant relationship between symptom severity and treatment adherence or between quality of sleep and treatment adherence in patients with depression. However, a significant positive relationship was found between negative symptoms and treatment adherence and a significant negative relationship between quality of sleep and treatment adherence in patients with schizophrenia. Conclusion: The present exploratory study revealed a positive relationship between symptom severity and treatment adherence and a negative relationship between quality of sleep and treatment adherence in patients with schizophrenia, but no significant relationships in patients with depression were found. Future studies are needed in order to gain a better understanding of possible risk factors related to treatment non-adherence.
\end{abstract}

Key words: Depression; quality of sleep; schizophrenia; symptom severity; treatment adherence

\section{INTRODUCTION}

Dropout is a serious problem in treating patients because treatment can only be effective if delivered adequately and completely. ${ }^{[1]}$ To date, very little is

Corresponding Author: Dr. Peggy Bosch, Centre for Cognition, Donders Institute for Brain, Cognition and Behaviour, Radboud University Niimegen, Postbus 9104, Montessorilaan 3, $6500 \mathrm{GL}$ Nijmegen, the Netherlands. E-mail: P.bosch@donders.ru.nl

\begin{tabular}{|l|l|}
\hline \multicolumn{3}{|c|}{ Access this article online } \\
\hline Quick Response Code: & Website: \\
\hline $\mathbf{D}$ & www.nnjournal.net \\
\cline { 1 - 2 } & DOI: 10.20517/2347-8659.2015.54 \\
\hline
\end{tabular}

known about possible risk factors that might predict patient dropout. ${ }^{[2]}$ Findings by Herman et al. ${ }^{[3]}$ indicate that early treatment dropout is an essential factor in determining treatment success and failure because in case of dropout, patients receive little or nothing of the intervention involved and, therefore, cannot

This is an open access article distributed under the terms of the Creative Commons Attribution-NonCommercial-ShareAlike 3.0 License, which allows others to remix, tweak, and build upon the work non-commercially, as long as the author is credited and the new creations are licensed under the identical terms.

For reprints contact: service@oaepublish.com

How to cite this article: Bosch P, Waberg J, van den Noort M, Staudte H, Lim S, Egger J. Symptom severity, quality of sleep, and treatment adherence among patients suffering from schizophrenia and depression. Neuroimmunol Neuroinflammation 2016;3:109-16.

Received: 11-12-2015; Accepted: 16-03-2016 
benefit from therapy. "Adherence to treatment" refers to the extent to which a patient's behavior conforms to the advice of health professionals. ${ }^{[4]}$ Although dropout and adherence to treatment are related issues, there are also important distinctions between these two concepts. ${ }^{[5]}$

Clinical psychologists have reported that, unfortunately, non-compliance with homework as part of cognitive-behavioral therapy is a common phenomenon in clinical practice. ${ }^{[6]}$ This is problematic because research has shown that homework compliance is positively associated with reduced symptom severity. ${ }^{[7]}$

Treatment non-adherence is a common problem in patients suffering from depression or schizophrenia. ${ }^{[8]}$ In cases of schizophrenia, poor adherence to medication and psychosocial treatment are prevalent and increase the probability of relapse and re-hospitalization. ${ }^{[9]}$ In their review of 39 studies published in English since 1980 and specifically examining risk factors for medication non-adherence, Lacro et al. ${ }^{[10]}$ pointed out that in those studies, the mean rate of non-adherence to medication for patients with schizophrenia was $41 \%$. Moreover, in their systematic review of 103 studies on adherence to treatment by patients with psychosis, Nosé et al. ${ }^{[11]}$ reported a mean rate of failure to keep scheduled follow-up appointments of $24 \%$ in patients with psychoses. Rates of treatment dropout are reported less often; the figures for this range from $20 \%$ to $56 \%$ in patients with schizophrenia. ${ }^{[12-14]}$ In patients with depression, the severity of the disorder has been associated with treatment dropout in some studies, ${ }^{[15]}$ but not in all. ${ }^{[16]}$ Agreeing with Last et al. ${ }^{[15]}$ Leserman $^{[17]}$ indicated that patients with depression were less likely to adhere to a treatment program than patients without depression and experienced worse outcomes in health.

Schizophrenia and depression are both seemingly dropout-prone disorders, ${ }^{[18,19]}$ but which specific factors can be related to the non-adherence to treatment in patients with either condition is arguable. An association between less severe psychiatric symptoms and better treatment adherence was found, ${ }^{[20]}$ and both the severity of the disease and the patient's attitude towards the prescribed medication were found to be related to adherence in patients with schizophrenia. ${ }^{[21]}$ In this case, the severity was found to correlate negatively with treatment adherence while the patient's attitude towards the prescribed medication was found to correlate positively with treatment adherence.

Also, a role for quality of sleep in treatment adherence of patients with schizophrenia or depression has been suggested. ${ }^{[22,23]}$ Phillips and colleagues, ${ }^{[23]}$ for instance, considered adherence to treatment to be a factor that correlated with sleep disturbance and depression. Their results showed that women with greater sleep disturbances also had a higher level of depressive symptoms and poor adherence to their medication regimen. According to these findings, ${ }^{[23]}$ not only the severity of the disorder but also suffering from sleep disturbances might be related to treatment non-adherence in patients with schizophrenia or depression. A possible role for quality of sleep in treatment adherence is further supported by the fact that both schizophrenia and depression are co-morbid with sleep disturbances. ${ }^{[24,25]}$

However, to date, not much research has been conducted on the specific factors that can be related to the nonadherence to treatment in patients with schizophrenia or depression. As a result, more research is needed on the possible factors suggested in the literature, symptom severity and quality of sleep, as being related to treatment non-adherence. The aim of the present study was, therefore, to investigate the possible relationships between symptom severities, quality of sleep, and treatment adherence further. Thereby, noting that treatment non-adherence limits the improvements in independent living, employment, and quality of life to a large degree in patients with schizophrenia or depression is important. ${ }^{[26]}$ First, patients with a more severe depression were hypothesized to have worse treatment adherence than patients with a less severe depression. Secondly, patients with schizophrenia who show more positive and negative symptoms were hypothesized to have worse treatment adherence than patients with schizophrenia who show fewer positive and negative symptoms. A final hypothesis was that patients who have a poor quality of sleep have worse treatment adherence than patients who have a good quality of sleep.

\section{METHODS}

\section{Setting and participants}

The participants in this study consisted of 17 female and 13 male adult patients with schizophrenia with an average age of $41(\mathrm{SD}=8.80)$ and 40 female and 18 male adult patients with depression with an average age of 45 (SD = 12.14). All participants were outpatients of the LVR-Klinik Bedburg-Hau in Germany and were diagnosed by their psychiatrist according to the 10th revision of the International Classification of Diseases and Related Health Problems (ICD-10). ${ }^{[27]}$ Note that in Germany, the ICD-10 ${ }^{[27]}$ is used instead of the Diagnostic and Statistical Manual 
of Mental Disorders, fifth edition (DSM-V). ${ }^{[28]}$ The inclusion criterion for all participants was that they be between 18 and 65 years of age, and the exclusion criteria for the patients with schizophrenia or depression were substance abuse, epilepsy, and other neurological disorders. Finally, all patients took part voluntarily without any inducement and signed the informed consent form.

\section{Material}

BDI-II

The Beck Depression Inventory-II (BDI-II) ${ }^{[29]}$ was used to assess depression severity. The BDI-II is a 21-item self-report inventory, and each item is rated on a 4-point scale (i.e. from 0 to 3 ). Thus, an individual's scoring range would lie between 0 and 63. The higher the total scores on the BDI-II, the more severe the depressive symptoms of the patients are.

\section{PANSS}

In addition, the Positive and Negative Syndrome Scale (PANSS) ${ }^{[30]}$ was used to measure the symptom severity of the patients with schizophrenia. The PANSS has to be filled in by a psychiatrist. It consists of three subscales: the positive scale, the negative scale and the general psychopathology scale. The scoring range for an individual is between 7 and 49 for the positive scale, 7 and 49 for the negative scale, and 16 and 112 for the general psychopathology scale; as a result, the total scores on the PANSS are between 30 and 210. The higher the total scores on the PANSS are, the more severe the positive and negative symptoms of the individuals with schizophrenia are.

\section{PSQI}

The Pittsburgh Sleep Quality Index (PSQI) ${ }^{[31]}$ was used to measure the subjective quality of sleep of the patients with schizophrenia or depression. The PSQI is a self-report inventory and measures sleep quality and patterns of sleep. It has seven separate domains: (1) "subjective sleep quality"; (2) "sleep latency"; (3) "sleep duration"; (4) "habitual sleep efficiency"; (5) "sleep disturbances"; (6) "use of sleep medication"; and (7) "daytime dysfunction over the last month". Individuals score all items on a Likert scale, ranging from 0 to 3 . As a result, the total scores on the PSQI are between 0 and 21, and the higher the total PSQI score is, the poorer the quality of sleep is. In daily clinical practice, often a cut-off score of $5^{[31]}$ is used, meaning that participants who score below 5 have a good quality of sleep and participants who score above 5 have a poor quality of sleep. In the present study, the total PSQI scores were used for further analyses.

\section{Sleep log}

As part of the treatment adherence measurements for the schizophrenic and depressive patients, ${ }^{[32]}$ the participants were asked to complete a sleep log for two weeks. It consisted of the following six sleep variables: (1) "total sleep time"; (2) "how many minutes awake during the night"; (3) "how many minutes awake before falling asleep"; (4) "how relaxing was your sleep"; (5) "did you feel exhausted"; (6) "how was your average performance level today". The sleep log was to be completed every morning just after awakening and every evening just before falling asleep ${ }^{[33]}$ Completing the sleep log took approximately $10 \mathrm{~min}$ a day, $140 \mathrm{~min}$ in total.

\section{Procedure}

Every participant in the group of patients with depression was asked to complete the BDI-II and the PSQI. The PANSS was completed by the patient's psychiatrist in the group of patients with schizophrenia, and these patients were also asked to complete the PSQI. All participants were asked to keep a sleep log and return it to their clinical psychologist at the end of the two weeks. At the end of the study, a debriefing was offered to all participants, in which they were individually informed of their test results. The study was approved by the local ethics committee (Ärztekammer Nordrhein, number: 2008331); moreover, the clinical trial has officially been registered under number NTR3132 at the Dutch Trial Register (see also http://www.trialregister.nl/ trialreg/admin/rctview.asp? TC = 3132). Finally, the study was performed in accordance with the Declaration of Helsinki (http://www.wma.net/ en/30publications/10policies/b3/).

\section{Design and statistics}

An experimental design was used in which the first categorical dependent variable was called "Absolute Treatment Adherence"; it was measured by using the returned sleep logs (i.e. if the patient returned the sleep log, the patient was considered as being treatment adherent $v s$. if the patient did not return the sleep log, the patient was considered as not being treatment adherent). The second continuous dependent variable was called "Degree of Treatment Adherence" because it consisted of the total number of days the participants had completed the sleep log, with 0 day meaning a very low degree of treatment adherence and 14 days meaning a very high degree of treatment adherence. In other words, "Absolute Treatment Adherence" showed whether a patient returned the sleep log or not while "Degree of Treatment Adherence" showed to what degree the patient was treatment adherent. Note that in the present study, a very narrow definition of the word "treatment" was used (e.g. completing and returning a sleep log) and did not include any pharmacological 


\begin{tabular}{|c|c|c|c|c|}
\hline Adherence & Instrument & Number & Mean & SD \\
\hline Non-adherent & $\begin{array}{l}\text { BDI-II } \\
\text { PSQI }\end{array}$ & $\begin{array}{l}21 \\
21\end{array}$ & $\begin{array}{c}20.81 \\
9.05\end{array}$ & $\begin{array}{r}12.21 \\
4.28\end{array}$ \\
\hline Adherent & $\begin{array}{l}\text { BDI-II } \\
\text { PSQI }\end{array}$ & $\begin{array}{l}37 \\
37\end{array}$ & $\begin{array}{c}19.24 \\
9.51\end{array}$ & $\begin{array}{c}11.28 \\
4.17\end{array}$ \\
\hline Total & $\begin{array}{l}\text { BDI-II } \\
\text { PSQI }\end{array}$ & $\begin{array}{l}58 \\
58\end{array}$ & $\begin{array}{c}19.81 \\
9.34\end{array}$ & $\begin{array}{c}11.54 \\
4.18\end{array}$ \\
\hline
\end{tabular}

All $P>0.05$; PSQI: pittsburgh sleep quality index; BDI-II: beck depression inventory-II

\begin{tabular}{lcccc}
\hline Table 2: The number of adherent and non-adherent \\
patients with schizophrenia and their mean scores and \\
standard deviations on the PANSS and the PSQI \\
Adherence & Instrument & Number & Mean & SD \\
\hline Non-adherent & PANSS-total & 16 & 67.81 & 19.56 \\
& PANSS-negative & 16 & $16.81^{*}$ & 6.76 \\
& PANSS-positive & 16 & 14.13 & 7.37 \\
& PANSS-psychopathology & 16 & 36.88 & 11.62 \\
Adherent & PSQI & 16 & 6.31 & 3.32 \\
& PANSS-total & 14 & 81.86 & 26.74 \\
& PANSS-negative & 14 & $23.43^{*}$ & 10.42 \\
& PANSS-positive & 14 & 20.71 & 26.44 \\
PANSS-psychopathology & 14 & 44.86 & 14.36 \\
Total & PSQI & 14 & 8.57 & 4.50 \\
& PANSS-total & 30 & 74.37 & 23.86 \\
& PANSS-negative & 30 & $19.90^{*}$ & 9.14 \\
& PANSS-positive & 30 & 17.20 & 18.78 \\
& PANSS-psychopathology & 30 & 40.60 & 13.37 \\
PSQI & 30 & 7.37 & 4.01 \\
\hline
\end{tabular}

${ }^{\star} P<0.05$; PANSS: positive and negative syndrome scale

interventions; neither did we use the sleep variables recorded in the sleep log to predict adherence. The scores on the BDI-II, the PANSS, and the PSQI served as "predictor" variables.

With SPSS version 22.0 $0^{[34]}$ a discriminant function analysis ${ }^{[35]}$ was conducted with "Absolute Treatment Adherence" as a categorical dependent variable and the scores on the BDI-II and PSQI as predictor variables for the group of patients with depression. For the group of patients with schizophrenia, a discriminant function analysis was performed with "Absolute Treatment Adherence" as a categorical dependent variable and the scores on the PANSS and PSQI as predictor variables. Furthermore, two multiple regression analyses ${ }^{[36]}$ were conducted with one analysis containing "Degree of Treatment Adherence" as the dependent variable and the score on the BDI-II, as well as the score on the PSQI, as independent variables for the group of patients with depression. In the second analysis, "Degree of Treatment Adherence" was used as the dependent variable, and the score on the PANSS, as well as the score on the PSQI, were used as independent variables for the group of patients with schizophrenia.

\section{RESULTS}

Absolute treatment adherence in the depression group For the group of patients with depression, a
Table 3: Results of the multiple regression analysis for variables predicting degree of treatment adherence

\begin{tabular}{lccc} 
Predictor & B & SEB & $\boldsymbol{\beta}$ \\
\hline Constant & 8.95 & 2.48 & - \\
BDI-II & -0.03 & 0.07 & -0.05 \\
PSQI & 0.13 & 0.21 & 0.08 \\
\hline
\end{tabular}

$R^{2}=0.01$; B: unstandardized multiple regression coefficient; SEB: standard error of multiple regression coefficient; $\beta$ : standardized multiple regression coefficient

Table 4: Results of the multiple regression analysis for variables predicting degree of treatment adherence

\begin{tabular}{lccc} 
Predictor & B & SEB & $\boldsymbol{\beta}$ \\
\hline Constant & -2.14 & 4.13 & - \\
PANSS-positive & 0.08 & 0.07 & 0.21 \\
PANSS-negative & 0.21 & 0.25 & 0.29 \\
PANSS-psychopathology & 0.01 & 0.32 & 0.02 \\
PANSS-total & -0.02 & 0.22 & -0.06 \\
PSQI & 0.65 & 0.31 & $0.39^{*}$ \\
\hline
\end{tabular}

$R^{2}=0.27{ }^{*} P<0.05$; B: unstandardized multiple regression coefficient; SEB: standard error of multiple regression coefficient; $\beta$ : standardized multiple regression coefficient

discriminant function analysis was conducted in order to identify patients who were adherent to treatment vs. those who were not; this was done using the patient's scores on the BDI-II and the PSQI as predictors. The discriminant function analysis explained $100 \%$ of the variance, canonical $R^{2}=0.01$ (Note that this does not mean that the discriminant function accounts for $100 \%$ of the variance in the response variable; rather, this means that it is the only discriminant function extracted for the analysis). ${ }^{[37]}$ The discriminant function analysis did not significantly differentiate the patients who were treatment-adherent from the ones who were not $\left[\Lambda=0.99, \chi^{2}(2)=0.43, P>0.05\right]$. Table 1 summarizes the descriptive statistics of the group of patients with depression.

\section{Absolute treatment adherence in the schizophrenia group}

In addition, a discriminant function analysis was conducted for the group of patients with schizophrenia. The discriminant model was used to identify patients who were adherent to treatment versus those who were not treatment-adherent by using the patient's scores on the PANSS (also divided into positive symptoms, negative symptoms, and general psychopathology) and on the PSQI as predictors. The discriminant function analysis explained $100 \%$ of the variance, canonical $R^{2}=0.26$. The discriminant function analysis did not significantly differentiate the patients who were treatmentadherent from the ones who were not $[\Lambda=0.74$, $\left.\chi^{2}(5)=7.67, P>0.05\right]$. The correlations between outcomes and the discriminant function revealed that the score on the PANSS-negative loaded highly onto the function $(r=0.67)$, followed by the score on the PSQI $(r=0.50)$. Table 2 summarizes the descriptive statistics of the group of patients 
with schizophrenia.

Variables predicting degree of treatment adherence in the depression group

For the group of patients with depression, a multiple regression analysis with "Degree of Treatment Adherence" as the dependent variable and the scores on the BDI-II and the PSQI as predictors was conducted. Note that the adherent depression group had a mean of 13.44 days (SD $=2.02$ days). Table 3 shows the results of the multiple regression analysis using the enter method for predicting “Degree of Treatment Adherence". The scores on the BDI-II and the PSQI were insignificant predictors.

Variables predicting degree of treatment adherence in the schizophrenia group

For the group of patients with schizophrenia, a multiple regression analysis was conducted with "Degree of Treatment Adherence" as the dependent variable and the scores on the PANSS-total (also split into PANSS-positive, PANSS-negative, and PANSS-general psychopathology) and on the PSQI as predictors. Note that the adherent schizophrenia group had a mean of 12.82 days ( $S D=2.43$ days). Table 4 shows the results of the multiple regression analysis using the enter method for predicting "Degree of Treatment Adherence". The scores on the PANSS-total, PANSS-positive, PANSS-negative, and PANSS-general psychopathology were insignificant predictors while the score on the PSQI was a significant predictor. Moreover, the score on the PANSS-negative and the score on the PSQI correlated significantly with "Degree of Treatment Adherence" (PANSS-negative: $r=0.31, P<0.05$; PSQI: $r=0.39$, $P<0.05)$.

\section{DISCUSSION}

The general results of our study showed that for the group of patients with depression, in contrast to our hypotheses, symptom severity and quality of sleep did not significantly predict whether a patient would be adherent to treatment or not; the severity of depression and the quality of sleep did not differ significantly between treatment adherent and non-adherent patients. For the group of patients with schizophrenia, in line with our hypotheses, a relationship was found between symptom severity and treatment adherence, as well as between quality of sleep and treatment adherence; however, the directions of those relationships were contrary to our expectations. Experiencing more severe negative symptoms was found to be significantly related to better treatment adherence. Moreover, quality of sleep did serve as a significant predictor of treatment adherence; however, in general, the patients with schizophrenia who reported a worse quality of sleep were more treatment-adherent than those who reported a better quality of sleep and not vice versa.

The surprising findings that no relationships were found between quality of sleep and treatment adherence and between symptom severity and treatment adherence in the depression group are opposed to the findings by Ong etal. ${ }^{[2]}$ who investigated the risk factors associated with dropping out of group cognitive-behavior therapy for insomnia and found that short sleep duration and elevated symptoms of depression at baseline could be especially associated with an increased risk of early therapy dropout. Also, in their study, Phillips et $a .^{[23]}$ reported that women with greater sleep disturbances had a higher level of depressive symptoms and poor adherence to their medication regimen. Noticeably, research that targets adherence to medication dominates research that targets adherence to treatment. Possibly, different risk factors may be related to these two forms of adherence, which might make a direct comparison between studies that target adherence to medication and studies that target adherence to treatment difficult. This suggestion remains to be verified. Overall, to date, only a few studies have been conducted on treatment non-adherence by patients with depression and its predictors, ${ }^{[38]}$ so further research is warranted.

Nevertheless, in the group of patients with schizophrenia in the present study, symptom severity and quality of sleep may be related to better treatment adherence. Steger et al. ${ }^{[39]}$ reported similar findings. The researchers assessed medication adherence in a sample of 216 patients with a first episode of psychosis; the assessments were done at program entry and three and six months later. They found an association between early resolution of negative symptoms and poor adherence. Patients whose positive symptoms had been resolved after three months of treatment did not show a change in adherence behavior compared to those whose symptoms persisted. On the contrary, early resolution of negative symptoms was significantly associated with less medication adherence compared to the patients whose negative symptoms persisted. Unfortunately, patients whose negative symptoms had been resolved but who were non-adherent experienced a worsening of both positive and negative symptoms at six month. Steger et al. ${ }^{[39]}$ concluded that patients who experience a rapid reduction of negative symptoms must be closely followed, as they are at high risk for non-adherence. As a possible 
explanation for these findings, the researchers stated that patients might associate reduction of negative symptoms with a return to normal functioning and, therefore, might decide that they no longer need medication. This assumption is supported by the finding of Quach et al. ${ }^{[40]}$ whose study indicated that a high level of functioning after one year of treatment was associated with non-adherence at year two.

However, opposing findings have also been reported in the treatment adherence literature on patients with schizophrenia. A study by Tattan and Creed ${ }^{[41]}$ regarding negative symptoms of schizophrenia and compliance with medication, for instance, found that patients with schizophrenia who had a poor medication compliance experienced a significantly greater severity of negative symptoms. Especially avolition, apathy, and alogia were related to poor compliance. The researchers presumed that patients who suffered from avolition and apathy would lack the motivation to regularly go to a satellite clinic. They also suggested that these patients might question the beneficial effects that the medication given at such clinics had had on their positive symptoms and might have instead focused on the limited effect that the medication had had on the negative symptoms from which they still suffer. Another point considered was that patients with alogia could possibly lack insight into their illness and, therefore, might not understand the importance of taking medication regularly. In sum, hitherto, as for depression, not much research has been conducted on the relationship between symptom severity and treatment adherence in patients with schizophrenia, and the results are conflicting. ${ }^{[39-41]}$ Clearly, negative symptoms can be related to adherence, but further research is needed to gain a better understanding of this relationship.

An interesting new finding of our study is the fact that poor quality of sleep significantly predicted better treatment adherence in the group of patients with schizophrenia. This finding contradicts the finding in the study by Ong et al. ${ }^{[2]}$ who reported that insomnia might be a risk factor for poor treatment adherence. To the authors' knowledge, this study was among the first to investigate a possible relationship between quality of sleep and treatment adherence in patients with schizophrenia; therefore, comparing this group of patients to another that has sleep disorders such as obstructive sleep apnoea and insomnia might be difficult. Possibly, the patients with a worse subjective quality of sleep were more treatment adherent because they could benefit from completing the sleep log and, hence, register their own sleeping patterns for a period of two weeks. Patients without sleeping problems might not understand the therapeutic use of registering their sleeping patterns and, therefore, might be more likely to not complete the sleep log.

In sum, treatment adherence appears to be a complex phenomenon and can only partly be associated with symptom severity and quality of sleep in patients with schizophrenia and patients with depression. Some studies indicate, for instance, that a weak therapeutic alliance and low insight might be related to poor adherence in patients with schizophrenia. ${ }^{[42]}$ Another study by Spiekermann et $a l^{[43]}$ stated that patients with schizophrenia who had stronger cognitive impairments showed lower adherence behavior compared to those without cognitive impairments.

The present study has several limitations that should be discussed in order to correctly interpret its results. One limitation of our study is the fact that all data were derived from self-report inventories, which have their strengths and weaknesses. ${ }^{[44]}$ However, the disadvantages, for instance, self-report measures being potentially biased by social desirability, ${ }^{[45]}$ might have negatively affected the reliability of the results. Moreover, future studies might vary in their means to measure treatment adherence. For instance, attendance at a psycho-education group for patients with schizophrenia ${ }^{[46]}$ could be additionally used to measure treatment adherence. Another limitation of our study is that a mixed sample of subjects, e.g., outpatients with schizophrenia and outpatients with depression, was used. Although we have presented our treatmentadherence results for the depression group and the schizophrenia group separately, having a larger sample of one patient group in order to investigate the possible relationships between symptom severity, quality of sleep, and treatment adherence would have been better because the main reasons related to non-adherence in patients with schizophrenia and those with depression may differ significantly. This is an important issue for future research. Finally, the exploratory nature of the analyses, as well as the cross-sectional study design, ${ }^{[47,48]}$ used in the present study should be mentioned as further limitations, making it impossible to generalisze the results.

In conclusion, the current exploratory study revealed a significant positive relationship between symptom severity and treatment adherence, as well as a significant negative relationship between quality of sleep and treatment adherence in patients with schizophrenia, but no significant relationships between symptom 
severity, quality of sleep, and treatment adherence were found in patients with depression. However, more research is needed to identify possible risk factors that can be related to treatment non-adherence to, in a second step, determine strategies to improve treatment adherence. Treatment non-adherence might be reduced, and at best prevented, if specific predictors can be related to the adherence behavior of psychiatric patients. Furthermore, identifying the characteristics of clinical patients who drop out of treatment could lead to improvements in the care these patients receive.

\section{Acknowledgments}

We thank Dr. Marie Brill; she is the director of the LVR-Klinik Bedburg-Hau and made this study possible. Moreover, we thank Prof. Gilles van Luijtelaar and Prof. Anton Coenen for their helpful comments.

\section{Financial support and sponsorship}

Nil.

\section{Conflicts of interest}

There are no conflicts of interest.

\section{REFERENCES}

1. Pinto-Meza A, Fernández A, Bruffaerts R, Alonso J, Kovess V, De Graaf R, de Girolamo G, Matschinger H, Haro JM. Dropping out of mental health treatment among patients with depression and anxiety by type of provider: results of the European Study of the Epidemiology of Mental Disorders. Soc Psychiatry Psychiatr Epidemiol 2011;46:273-80.

2. Ong JC, Kuo TF, Manber R. Who is at risk for dropout from group cognitive-behavior therapy for insomnia? J Psychosom Res 2008;64:419-25.

3. Herman S, Blumenthal JA, Babyak M, Khatri P, Craighead WE, Krishnan KR, Doraiswamy PM. Exercise therapy for depression in middle-aged and older adults: predictors of early dropout and treatment failure. Health Psychol 2002;21:553-63.

4. Haynes RB. Determinants of compliance: the disease and the mechanics of treatment. In: Haynes RB, Taylor DW, Sacket DL, editors. Compliance in health care. Baltimore: Johns Hopkins University Press; 1979. p. 49-62.

5. Miller R, Ream G, McCormack J, Gunduz-Bruce H, Sevy $\mathrm{S}$, Robinson D. A prospective study of cannabis use as a risk factor for non-adherence and treatment dropout in first-episode schizophrenia. Schizophr Res 2009;113:138-44.

6. Helbig S, Fehm L. Problems with homework in CBT: rare exception or rather frequent? Behav Cogn Psychoth 2004;32:291-301.

7. Kazantzis N. Power to detect homework effects in psychotherapy outcome research. J Consult Clin Psychol 2000;68:166-70.

8. Startup M, Wilding N, Startup S. Patient treatment adherence in cognitive behaviour therapy for acute psychosis: the role of recovery style and working alliance. Behav Cogn Psychoth 2006;34:191-9.

9. McCann TV, Boardman G, Clark E, Lu S. Risk profiles for nonadherence to antipsychotic medications. J Psychiatr Ment Health Nurs 2008;15:622-9.

10. Lacro JP, Dunn LB, Dolder CR, Leckband SG, Jeste DV. Prevalence of and risk factors for medication nonadherence in patients with schizophrenia: a comprehensive review of recent literature. J Clin Psychiatry 2002;63:892-909.

11. Nosé M, Barbui C, Tansella M. How often do patients with psychosis fail to adhere to treatment programmes? A systematic review. Psychol Med 2003;33:1149-60.

12. Gaebel W, Jänner M, Frommann N, Pietzcker A, Köpcke W, Linden M, Müller P, Müller-Spahn F, Tegeler J. First vs. multiple episode schizophrenia: two-year outcome of intermittent and maintenance medication strategies. Schizophr Res 2002;53:145-59.

13. Kamali M, Kelly BD, Clarke M, Browne S, Gervin M, Kinsella A, Lane A, Larkin C, O'Callaghan E. A prospective evaluation of adherence to medication in first episode schizophrenia. Eur Psychiatry 2006;21:29-33.

14. Linden M, Godemann F, Gaebel W, Köpke W, Müller P, MüllerSpahn F, Pietzcker A, Tegeler J. A prospective study of factors influencing adherence to a continuous neuroleptic treatment program in schizophrenia patients during 2 years. Schizophr Bull 2001;27:585-96.

15. Last CG, Thase ME, Hersen M, Bellack AS, Himmelhoch JM. Patterns of attrition for psychosocial and pharmacologic treatments of depression. J Clin Psychiatry 1985;46:361-6.

16. Tedlow JR, Fava M, Uebelacker LA, Alpert JE, Nierenberg AA, Rosenbaum JF. Are study dropouts different from completers? Biol Psychiatry 1996;40:668-70.

17. Leserman J. HIV disease progression: Depression, stress, and possible mechanisms. Biol Psychiatry 2003;54:295-306.

18. De Crescenzo F, Perelli F, Armando M, Vicari S. Selective serotonin reuptake inhibitors (SSRIS) for post-partum depression (PPD): a systematic review of randomized clinical trials. $J$ Affect Disord 2014;152-154:39-44.

19. Gaebel W, Möller HJ, Buchkremer G, Ohmann C, Riesbeck M, Wölwer W, Von Wilmsdorff M, Bottlender R, Klingberg S. Pharmacological long-term treatment strategies in first episode schizophrenia: study design and preliminary results of an ongoing RCT within the German Research Network on Schizophrenia. Eur Arch Psychiatry Clin Neurosci 2004;254:129-40.

20. Tsang HW, Fung KM, Chung RC. Self-stigma and stages of change as predictors of treatment adherence of individuals with schizophrenia. Psychiatry Res 2010;180:10-5.

21. Yang J, Ko YH, Paik JW, Lee MS, Han C, Joe SH, Jung IK, Jung HG, Kim SH. Symptom severity and attitudes toward medication: impacts on adherence in outpatients with schizophrenia. Schizophr Res 2012;134:226-31.

22. Afonso P, Brissos S, Cañas F, Bobes J, Bernardo-Fernandez I. Treatment adherence and quality of sleep in schizophrenia outpatients. Int J Psychiatry Clin Pract 2014;18:70-6.

23. Phillips KD, Moneyham L, Murdaugh C, Boyd MR, Tavakoli A, Jackson K, Vyavaharkar M. Sleep disturbance and depression as barriers to adherence. Clin Nurs Res 2005;14:273-93.

24. Cohrs S. Sleep disturbances in patients with schizophrenia: impact and effect of antipsychotics. CNS Drugs 2008;22:939-62.

25. Szklo-Coxe M, Young T, Finn L, Mignot E. Depression: relationships to sleep paralysis and other sleep disturbances in a community sample. J Sleep Res 2007;16:297-312.

26. Glynn SM. Psychiatric rehabilitation in schizophrenia: advances and challenges. Clin Neurosci Res 2003;3:23-33.

27. World Health Organization. The ICD-10 Classification of Mental and Behavioural Disorders: Clinical Descriptions and Diagnostic Guidelines. Geneva, CH: World Health Organization Press; 1992.

28. American Psychiatric Association. Diagnostic and Statistical Manual of Mental Disorders. 5th ed. Arlington, VA: American Psychiatric Publishing; 2013.

29. Beck AT, Steer RA, Brown GK. Manual for the Beck Depression Inventory-II. San Antonio, TX: Psychological Corporation; 1996.

30. Kay SR, Fiszbein A, Opler LA. The Positive and Negative Syndrome Scale (PANSS) for schizophrenia. Schizophr Bull 1987;13:261-76.

31. Buysse DJ, Reynolds CF, Monk TH, Berman SR, Kupfer DJ. The Pittsburgh sleep quality index: a new instrument for psychiatric practice and research. Psychiatry Res 1989;28:193-213.

32. Kyle SD, Morgan K, Spiegelhalder K, Espie CA. No pain, no gain: an exploratory within-subjects mixed-methods evaluation of the patient experience of sleep restriction therapy (SRT) for insomnia. Sleep Med 2011;12:735-47.

33. Fischer J, Mayer G, Peter JH, Riemann D, Sitter H, editors. Nicht-erholsamer Schlaf. Non-relaxing sleep. Guideline, "S2" of the German Society of Sleep Research and Sleep Medicine. [In German] Berlin: Wissenschafts-Verlag; 2002.

34. IBM Corp. IBM SPSS Statistics for Windows, Version 22.0. Armonk, NY: IBM Corp; 2013.

35. McLachan GJ, editor. Discriminant analysis and statistical pattern recognition. New York, NY: Wiley; 1992.

36. Sax PE, Meyers JL, Mugavero M, Davis KL. Adherence to 
antiretroviral treatment and correlation with risk of hospitalization among commercially insured HIV patients in the United States. PLoS One 2012;7:e31591.

37. Denis DJ. Applied univariate, bivariate, and multivariate statistics. Hoboken, NJ: John Wiley and Sons; 2015.

38. Stein-Shvachman I, Karpas DS, Werner P. Depression treatment non-adherence and its psychosocial predictors: differences between young and older adults? Aging Dis 2013;4:329-36.

39. Steger KA, Cassidy C, Rabinovitch M, Joober R, Malla A. Impact of symptom resolution on medication adherence in first episode psychosis. Psychiatry Res 2012;196:45-51.

40. Quach PL, Mors O, Christensen T $\varnothing$, Krarup G, Jørgensen P, Bertelsen M, Jeppesen P, Petersen L, Thorup A, Nordentoft M. Predictors of poor adherence to medication among patients with first episode schizophrenia spectrum disorder. Early Interv Psychiatry 2009;3:66-74.

41. Tattan TM, Creed FH. Negative symptoms of schizophrenia and compliance with medication. Schizophr Bull 2001;27:149-55.

42. Misdrahi D, Petit M, Blanc O, Bayle F, Llorca PM. The influence of therapeutic alliance and insight on medication adherence in schizophrenia. Nord J Psychiatry 2012;66:49-54.

43. Spiekermann A, Schulz M, Behrens J, Driessen M, Rahn E, Beblo T. Adherence and cognitive performance in schizophrenia. Fortschr Neurol Psychiatr 2011;79:73-82.

44. Paulhus DL, Vazire S. The self-report method. In: Robins RW, Fraley RC, Krueger RF, editors. Handbook of research methods in personality psychology. New York, NY: Guilford; 2007. p. 224-39.

45. van de Mortel TF. Faking it: social desirability response bias in self-report research. Aust $J$ Adv Nurs 2008;25:40-8

46. Pitschel-Walz G, Bäuml J, Bender W, Engel RR, Wagner M, Kissling W. Psychoeducation and compliance in the treatment of schizophrenia: results of the Munich Psychosis Information Project Study. J Clin Psychiatry 2006;67:443-52.

47. Levin KA. Study design III: cross-sectional studies. Evid Based Dent 2006;7:24-5.

48. Mann CJ. Observational research methods. Research design II: cohort, cross sectional, and case-control studies. Emerg Med $J$ 2003;20:54-60 